\title{
Compendial Guidance for Particles
}

\author{
D. Scott Aldrich ${ }^{1}$ \\ ${ }^{1 .}$ Ultramikro LLC, Richland, MI, USA
}

The primary pharmacopeias for global pharmaceutical commerce are the United States Pharmacopeia (USP), European Pharmacopeia (EP or Ph. Eur.), and the Japanese Pharmacopeia (JP). Many of the general chapters and Monographs in these compendia are harmonized through the Pharmacopeial Discussion Group (PDG).

The USP provides guidance for all forms of pharmaceutical products for distribution in the United States. Broadly, cGMP regulations direct product design and assembly to provide final product cleanliness and stability to exclude foreign matter and minimize subvisible particle content. Specifically, there is further guidance in two general categories of particulate matter considered in several USP chapters as visible particles, based upon human detection, and subvisible particle content, based upon instrumental determination. The particle load must be considered in total in order for effective remediation and product improvement [1]. Recent revisions of USP general chapters and informational chapters have provided new or revisions of key definitions, techniques, methods and limits.

USP guidance for particulate matter content is comprehensively addressed in chapters $<1>$ Injections, $<788>$ Particulate Matter Determination [2], <1788> Methods for Determination of Particulate Matter in Injections [3], and $<789>$ Ophthalmic Solutions. For ophthalmic products, expansion of consideration has been drafted in a new $<771>$ Ophthalmic Products - Quality Tests, which is a significant alteration of current $<771>$ Ophthalmic Ointments. This new version of the ophthalmic general chapter considers product dose form descriptions, universal product quality tests and general particle quality expectations for all forms of ophthalmic products. Chapter $<751>$ Metal Particles in Ophthalmic Ointments has been removed since the new chapter provides general particle control guidance.

Further, new chapters <787> Subvisible Particulate Matter in Therapeutic Protein Injections and $<1787>$ Measurement of Subvisible Particulate Matter in Therapeutic Protein Injections provide guidance for analyzing the particle content of protein therapeutic products. With the interest in the effects of uncontrolled aggregation of protein therapeutic products upon immunogenicity, these new USP chapters provide alternate methods for determination of the inherent particulate character, as well as quantitation of unwanted particle types.

Historically, we concentrated on control of extraneous particulate matter. Recent initiatives in guidance revision focus on the proper development and physical stability of the dosage forms as well. USP has vigorously reviewed and innovated in the recent expert committee cycles, to provide solid guidance in product quality expectations.

There are broad terms for the description and control of particulate matter, in any size, consistent across all of the chapters:

Extrinsic material is unwanted, additive, foreign, and unchanging, not part of the formulation, package, or assembly process. 
Intrinsic material originates from the product and process - formulation, package, and commercial assembly steps, as both additive material, from contact, exposure or handling or as the result of product change and instability, such as from extracted moieties.

Inherent - Materials that are expected, in fact part of the product design constitute the inherent property of the formulation. They are acceptable characteristics of the product, evaluated and controlled from Development into commercial production. Especially for dosage forms such as emulsions, suspensions and protein aggregates, the material is well-known and predictable.

For all pharmaceutical organizations, their contract manufacturing partners, the analytical labs within the firms or contracted to provide service, these changes and broadened awareness of particle content are important. The expectation of the compendial organizations and Regulatory agencies is deep understanding of the product and ultimate control of the commercial production to ensure cleanliness, stability and purity. Systems of detection and investigation for all process streams and final assembly of the product are expected and necessary. Life Cycle management of all products is expected [1].

For microscopists, spectroscopists and materials scientists, our challenge is clear; apply our disciplines in a coordinated, integrated effort in the support of designing, assembly and monitoring product quality from Time-of-Release through End-of-Shelf Life. While the historical particle determination methods for injections have been light obscuration and membrane microscopy [2] [3], guidance for the investigation of the particle nature in protein products has provided a variety of options for techniques and case history examples [4].

\section{References:}

[1] D.S. Aldrich, R.T. Cherris and J.G. Shabushnig "Visual Inspection and Particulate Control”, DHI Publications, River Grove IL.

[2] United States Pharmacopeia (USP) (2016) General Chapter $<788>$ Particles in Injections. USP 39/NF 34. www.usp.org.

[3] United States Pharmacopeia (USP) (2016) Information Chapter $<1788>$ Methods for Determination of Particulate Matter in Injections. USP 39/NF 34. www.usp.org.

[4] United States Pharmacopeia (USP) (2016) General Chapter $<1787>$ Measurement of Subvisible Particulate Matter in Therapeutic Protein Injections. 39/NF 34. www.usp.org. 